\title{
Illumination change underneath bridge influences driver behavior
}

\author{
Xudong $\mathrm{Li}^{1, \mathrm{a}^{*}}$, Xiaowei Lian ${ }^{2, \mathrm{~b}}$ and Fujiu liu ${ }^{3, \mathrm{c}}$ \\ ${ }^{1,2,3}$ China Automotive Technology \& Research Center, \\ No.3 Boxing Six Rd, Economic-Technological Development Area, Beijing,China \\ a *lixudong@catarc.ac.cn, ${ }^{\text {b }}$ lianxiaowei@catarc.ac.cn, ${ }^{\mathrm{c}}$ liufuju@catarc.ac.cn
}

\begin{abstract}
Keywords: illumination change, visual space, driver behavior, natural driving study.
Abstract. Illumination change can influence driver behavior. Especially, glare light can make driver blind and lost vision, caused accidents happen. Driving behavior is a complex psychological activity process, and many researchers want to find common driving behavior characteristics. This paper studied driving behaviors, found in big cities, like Beijing, bridge is one factor can cause illumination change, data shown that mostly driver would change their driver behaviors instinctive, vehicle acceleration changed in a regular wave. Degree of illumination change will cause different acceleration variations. This paper discussed the reason how this phenomenon happen.
\end{abstract}

\section{Introduction}

With the rapid development of cities in China, especially, in big cities, Beijing and Shanghai, Interchanges were built to ease the traffic pressure. Interchanges need bridges to divide vehicles to different spaces and keep vehicle drive without disturbance. But bridges keep out light illuminate the road underneath and light change obviously when drive through.

As is known to all, vision, important perception ability, provide more than $80 \%$ information in our daily life, drivers need enough vision information to make correct decision and keep drive safety. In 1926, American physicist Holladay first puts forward the concept of glare, and he pointed that glare light make an equivalent veiling luminance on human eyes that reduce the ability of observation important objects[1]. Glare occur in middle vision that is between photopic vision and scotopic vision and the brightness is between $0.001 \sim 3 \mathrm{~cd} / \mathrm{m}^{2}$. Internationally, the glare can be divided into uncomfortable glare and disability glare, uncomfortable glare cause driver uncomfortable, but disability glare cause driver vision ability reduce[2]. Cells of the human eye is sensitive to shortwave with rods and short wavelength cones, these two kinds of cell have different sensitive to brightness and darkness. Experiment shown that glare light mainly influence columnar cells[3].

Normal driving behavior is a complex psychological activity process[4], many factors influence driving behavior, some behaviors are the habits of drivers but some are not. We are looking for common driving behaviors that maybe drivers don't mention them. These behaviors can inflect human instincts, and may cause accidents happen.

This paper studies the driving behaviors of driving underneath the bridges. Different from tunnel, illumination change through bridge is quick and not obvious. Time for driver adjustment is small, so there are evidently acceleration changes. This phenomenon can make a mistake for some advanced automotive active technology products, and we study why this wave happen.

\section{Vision and driver cognitive response pattern}

Vision is divided into static vision and dynamic vision. Static vision is the range of vision you can see when your head is fixed and eye forward. Dynamic vision refers to the range of vision that only the head fixed, eyeball freedom. The front view is main view for driver that is the view through the wind glass, this view determines the main information for drivers.

Adaptive problem is a common problem. Time is needed to adapt when human go to darkness from brightness or to brightness from darkness. In usually, it need more time to adapt darkness, but when divers drive in road that illumination is in a sharp change of light and shade. Because of adaptive time delay, it will cause vision reduction and accidents happen. 
Driver cognitive response pattern is consisted of three parts: apperceive stage, judge stage, response stage. Apperceive stage is collecting information about driving environment, visual information play main role in this stage. Judge stage is analysis of information depend on driver experience. Response stage is doing the action. Statistics shown that $48.1 \%$ of accidents happen because of mistake in apperceive stage, $36 \%$ in judge stage and only $7.8 \%$ in response stage.

\section{Experiments and working condition filter}

We used video camera and OBD recorder to do our experiments. Three cameras were used. One camera was set to collect drivers face information. One camera was set to collect front view and last camera was set to collect backward view, GPS data, speed data and acceleration data were collect by camera hardware. OBD recorder collected data like brake position, gear position and throttle pedal position, as shown in figure 1.

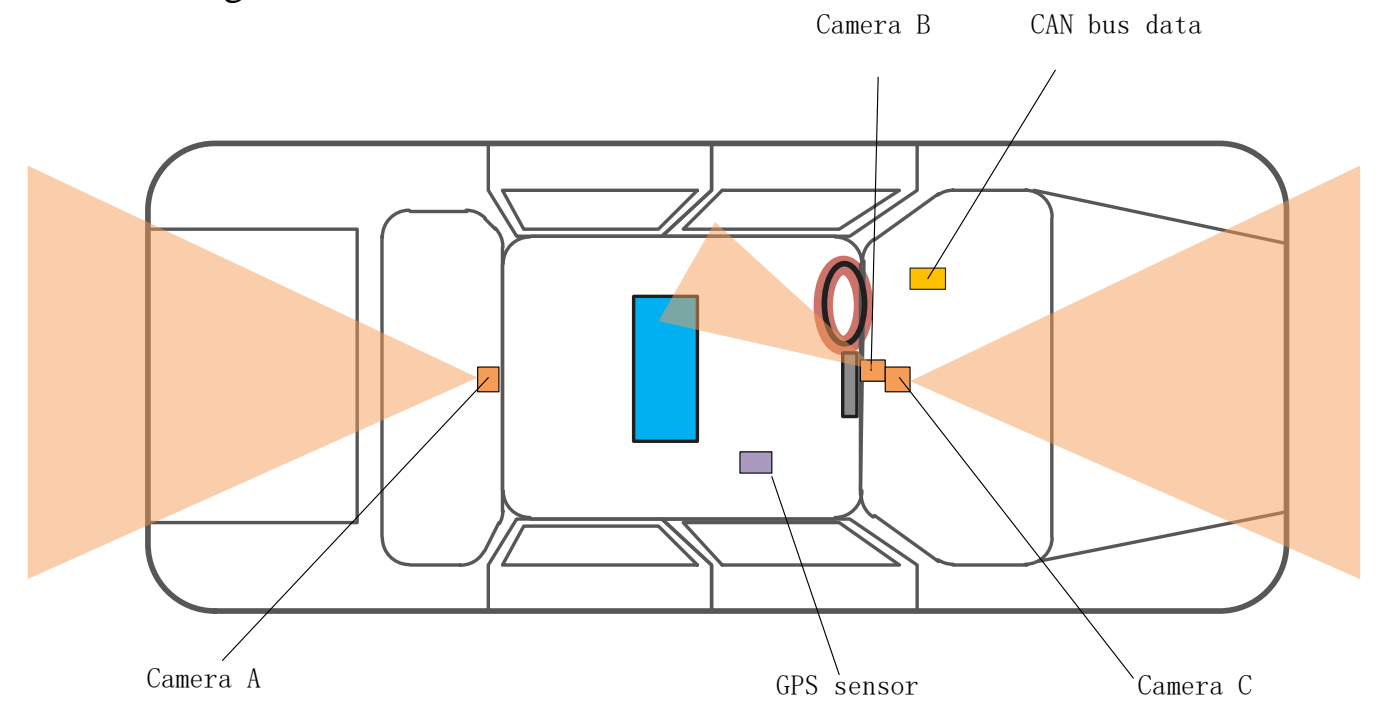

Figure 1, Sensors installation position

Nine drivers driving behaviors data were collected. Most of them drove in Beijing and Jinan. The primary requirement for condition selected is the driving is in a freedom traffic flow. There is no other factor to influence driver except bridge. Start time, end time, peak acceleration, valley acceleration, break action or throttle pedal position were collected to calculate the drivers behaviors.64 times underneath the bridges were chosen through the video by manual.

\section{Analysis and result}

All the 64 times underneath bridges had the similar waves, including 2 times at night. The average time used is $5.97 \mathrm{~s}, 62.5 \%$ of time used is less than $7 \mathrm{~s}$. The average time is small and not enough for driver to adapt. With the vehicle speed is high, the time used is small. Time used data was shown in figure 2 . 


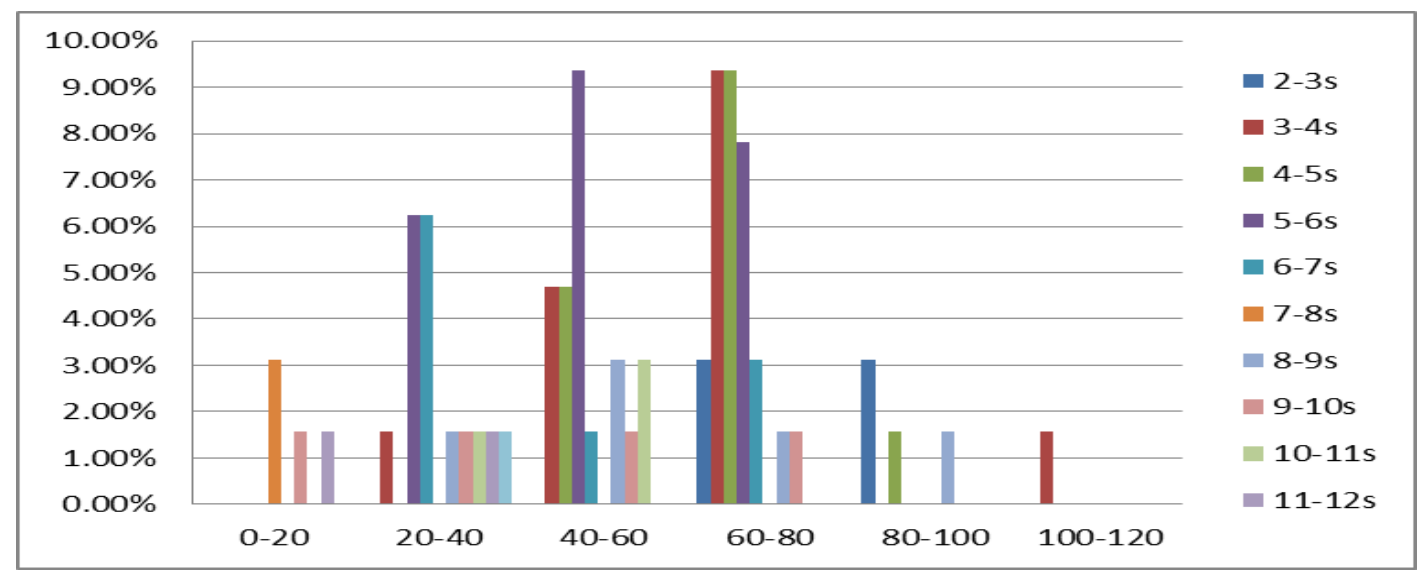

Firgure 2, Time used for crossing bridge

$56.25 \%$ of vehicle speed after bridge is higher than before, $-5 \sim 5 \mathrm{~km} / \mathrm{h}$ speed change is the biggest parts. There are two kinds of wave occurred most frequent. One is peak wave front and then gallery wave, the other one is gallery wave front. But peak wave and gallery wave adjoin with each other.

Two kinds of wave were shown in figure 3.

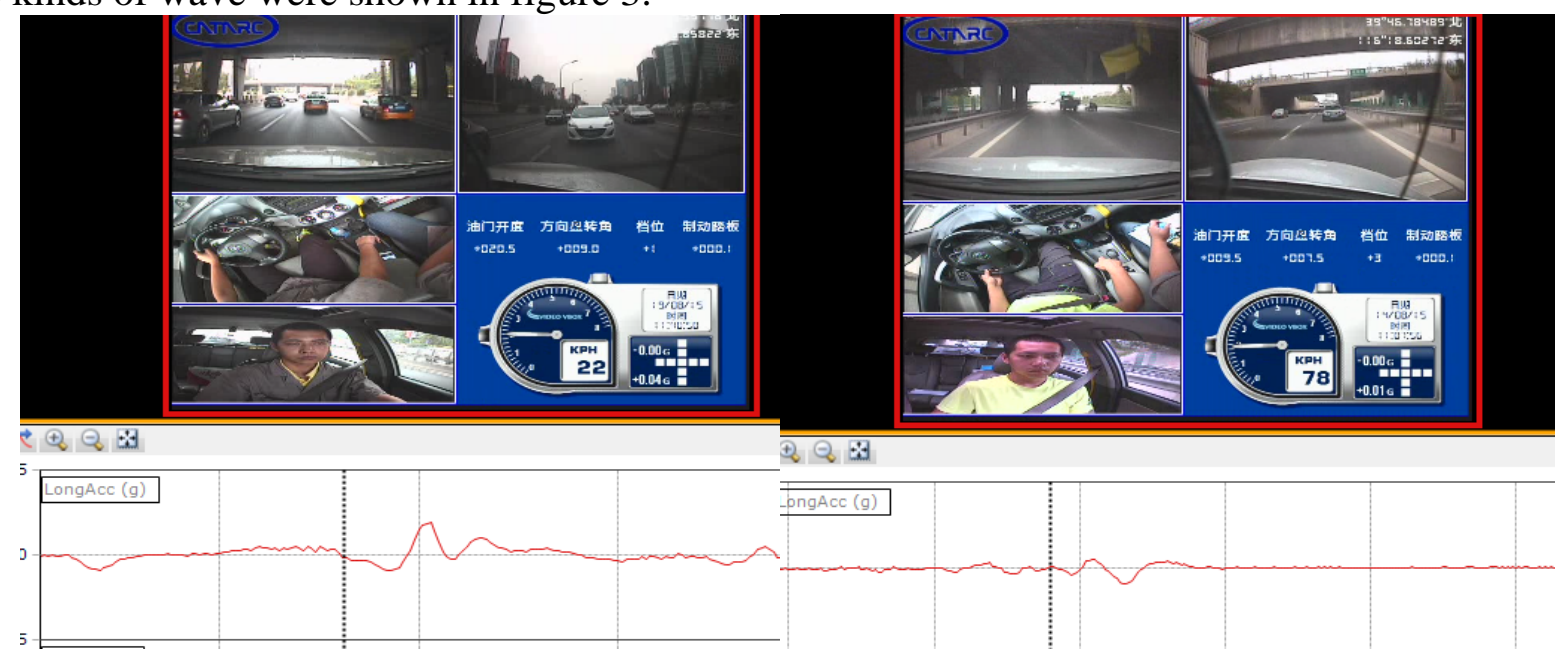

Figure 3, Two kinds of frequent waves

The time between peak and gallery is small, $53.13 \%$ is less than $1 \mathrm{~s}, 35.94 \%$ is between $1 \mathrm{~s}$ and $2 \mathrm{~s}$, the average acceleration change is $0.73 \mathrm{~g}$, it was a big change and can be found easily. As we know, some advanced safety equipment would alert when acceleration change is higher than $0.7 \mathrm{~g}$, so this situation would make the system mistake alert.

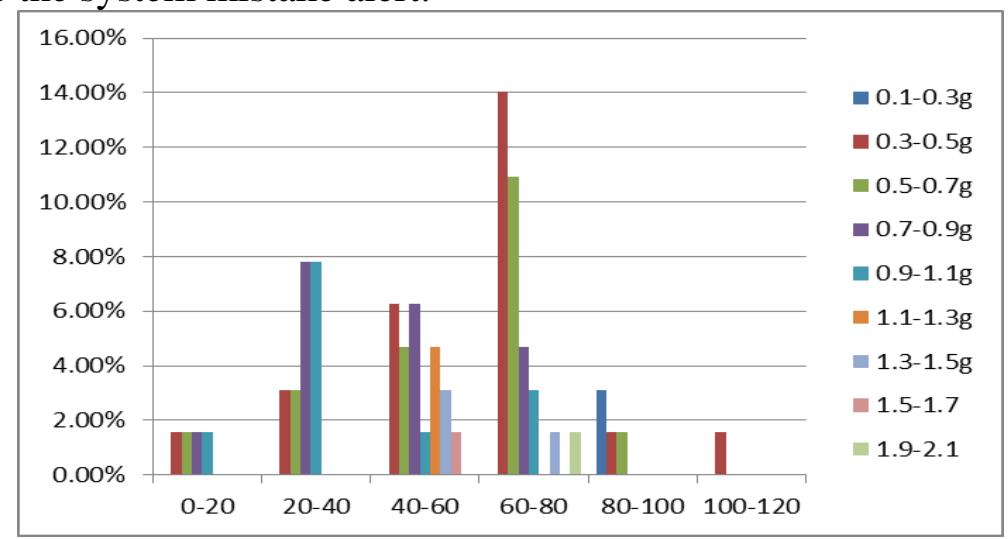

Figure 4, Vehicle speed and acceleration change

Figure 4 showed the relationship between vehicle speed and acceleration change. When vehicle speed is low, acceleration change range is big, but when vehicle speed is high, acceleration change is small, and the value of change is also small. 


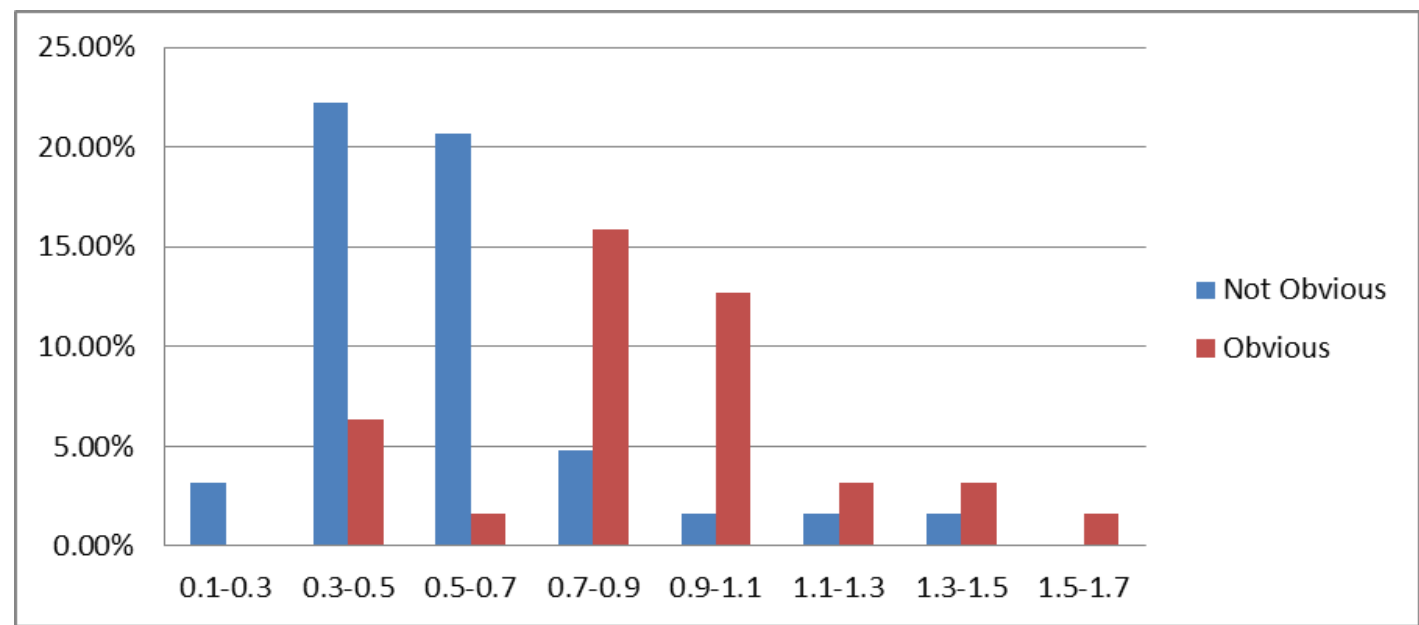

Figure 5, Illumination-acceleration change

Figure 5 showed that when illumination change is not obvious, acceleration change is small, but when illumination change is obvious, acceleration change is large.

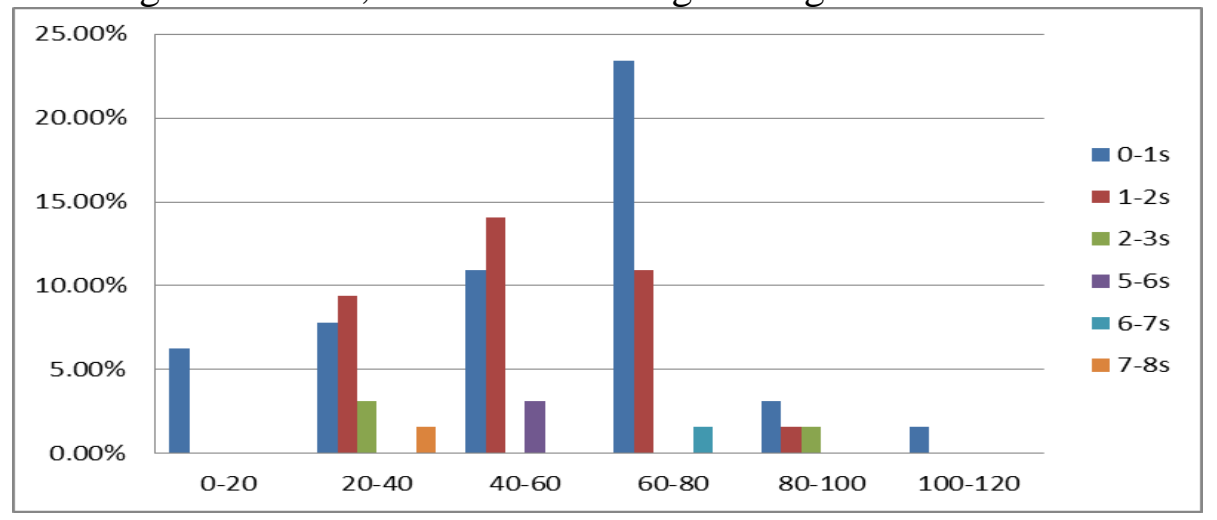

Figure 6, Time between acceleration peak and gallery

Figure 6 showed time between acceleration peak and gallery. High speed cross bridge , change time is small. With figure 5, it found that illumination change underneath bridge influence driving behavior need two factors, time and illumination change.

Illumination change influence driver vision, when driver went underneath bridge, darkness would affect driver mind, and when went out bridge, brightness would cause driver vision reduction and could not see front clearly, but when speed was high, time for driver adapt darkness is small, when driver went out bridge, eyes still keep the vision before, then illumination could not affect driver behavior evidently.

\section{Conclusion}

Illumination influence driving behaviors, this paper used driver behavior data, found except tunnel, bridge also can make a evidently illumination change and influence drivers behaviors. Acceleration waves showed a peak wave and a gallery wave adjoined when cross underneath bridge, the average acceleration change is $0.73 \mathrm{~g}$,this value is big enough to let some safety equipment alert. Two factors influenced the acceleration change: illumination change degree and time cross the bridge. Acceleration change is high when illumination change degree is obvious, but when time is small enough, acceleration would be small.

\section{References}

[1] Liandong $\mathrm{Yu}$, Qiaoyun Liu, Suhong Yu, Hongtao Wang, Study the formation mechanism of disability glare, Journal of hefei university of technology (natural science edition), 2005,08:866-868. 
[2] Patmos. P .GLARE AND TUNNEL ENTRANCE LIGHTING: EFFECTS OF STRAYLIGHT FROM EYE, ATMOSPHERE AND WINDSCREEN. CIE-journal. Jun 1984.

[3] Qi Long, Yandan Lin, Hong Shao, Dawei Chen, The middle visual analyses, China light \& lighting, 2002(9).

[4] Enliang Zhang, Guiping Xiao, Lei Nie, The influence of traffic environment on the driver's psychological analysis and strategy study, Road traffic technology, 2006(11)

[5] Xicheng Liu, Study on the Influence of Glare on Drivers' Visual Works at Night, College of Architecture and Urban Planning Chongqing University, 2007,3. 